\title{
The Impact of Skin Disease on Quality of Life in Rural Communities of Ghana
}

\author{
Caroline W. Laggis, MD1, Aaron M. Secrest, MD, $\mathrm{PhD}^{1,2}$, Martin Agyei, $\mathrm{MD}^{3}$, Sam Simister, $\mathrm{BS}^{4}$, \\ Andera N. Davis, $\mathrm{PhD}^{5}$, Ty Dickerson, MD, MPH${ }^{4}$, Jamie L.W. Rhoads, MD, $\mathrm{MS}^{1}$ \\ ${ }^{1}$ Department of Dermatology, University of Utah, Salt Lake City, UT, USA \\ ${ }^{2}$ Department of Population Health Sciences, University of Utah, Salt Lake City, UT, USA \\ ${ }^{3}$ Komfo Anokye Teaching Hospital (KATH), Kumasi, Ghana \\ ${ }^{4}$ School of Medicine, University of Utah, Salt Lake City, UT, USA \\ ${ }^{5}$ Department of Geography, University of Utah, Salt Lake City, UT, USA
}

\section{ABSTRACT}

Background: Skin disease is the fourth-leading cause of non-fatal disability worldwide. Granular data are limited on the skin disease burden in underserved populations.

Objectives: To describe the skin disease burden among adults in rural Ghana.

Methods: In this observational study, 230 adults in rural Ghana were surveyed using the Dermatology Life Quality Index (DLQI). For those reporting a skin problem in the previous week $(n=117)$ and who had skin examination performed by a dermatology resident and/or local dermatologist $(n=98)$, prevalence and univariate comparisons were calculated.

Results: $51 \%(117 / 230)$ of participants reported a skin problem in the previous week with $36 \%(42 / 117)$ reporting at least a moderate impact on quality of life (QoL). Factors associated with a higher QoL impact included female gender $(p=0.01)$ and living further from the city center $(p=0.02)$. The most common dermatologic diagnoses for those with skin examination performed included acne, bacterial infection, and pruritus. QoL was most impacted (highest average DLQI scores) for those with scabies. Diagnoses were categorized by the level of treatment or medical expertise that would be required had the participant presented to a clinic. $80 \%(78 / 98)$ of diagnoses rendered were potentially manageable with counseling or topical medication.

Limitations: The studied cohort was obtained via convenience sampling. The DLQI has not yet been validated in this population.

Conclusions: Much of the QoL impact from skin disease among adults in rural Ghana resulted from diagnoses that are manageable with counseling and topical medication. Better access to basic health care and more dermatologic education among community health providers would address much of the skin disease burden in these communities. Future studies should examine best practices for addressing unmet dermatologic needs of this and other comparable populations in underserved communities.

\section{INTRODUCTION}

The 2015 Global Burden of Disease (GBD) reported that skin and subcutaneous disorders are the fourth leading cause of non-fatal disease burden worldwide, with dermatitis causing the greatest burden. ${ }^{1}$ The GBD provides valuable insight into the burden of skin disease over time and by September 2020 Volume 4 Issue 5 
geographic location, however, more granular data from rural communities in low- and middle-income countries (LMICs) are limited. ${ }^{2}$ Currently in Ghana, fewer than 25 dermatologists are practicing ${ }^{3}$ in a country with a growing population of 28 million. ${ }^{4}$ In a referral center in urban Accra, Ghana, the most commonly-diagnosed skin conditions in adults were infections, dermatitis, acne vulgaris, and scabies. $^{3}$ Most Ghanaian dermatologists, and general physicians, practice in urban centers, leaving residents living in rural communities to rely on the intermittent presence of physician assistants at clinic outposts for their health care needs. ${ }^{5}$

The Dermatology Life Quality Index (DLQI) is a 10-question dermatology-specific assessment of quality of life (QoL) that has been translated and linguistically-validated in the Ghanaian language of Twi. ${ }^{7}$ DLQI has been used in previous studies in Africa, but primarily in disease-specific QoL studies. ${ }^{8-13}$ A large South African study, using a modified DLQI, explored the disability of skin disease in adults presenting to a dermatology clinic and found that social class and language influenced the DLQI scores. ${ }^{14}$

The current study aims to better understand the QoL impact of skin disease in rural communities of the Ashanti Region of Ghana in West Africa. Looking at dermatologic disease burden in specific communities brings attention to the spectrum of this burden across the geographic and economic spectrum. By better describing the burden of skin disease as it relates to demographic and diseaserelated factors in Ghana, we are contributing to a collaborative response towards understanding and reducing the burden of skin disease in this and comparable LMICs.

\section{METHODS}

The current project is a collaborative effort between the University of Utah's Division of Public Health (DPH) and Department of Dermatology with the Komfo Anokye Teaching Hospital (KATH), Kwame Nkrumah University of Science and Technology (KNUST), and the Atwima Nwabiagya North District of the Ashanti Region near Kumasi, Ghana (Figure 1). For the last decade, teams from University of Utah's DPH have collaborated with KATH and KNUST to conduct epidemiological studies in this region, steadily building a relationship of trust and comradery with the leaders and community members of the District. This study was approved by both the Committee on Human Research, Publications, and Ethics of KNUST and KATH and the University of Utah Institutional Review Board (IRB 00111394).

We developed a questionnaire to gather demographic data from adult participants. This questionnaire was tested for face validity and readability, then, along with the DLQI, was uploaded to digital tablets using Ona software in both Twi and English. The answer options to all DLQI questions are: (0) not at all; (1) a little; (2) a lot; and (3) very much. The final score (range 0-30) reflects the impact of an individual's skin disease on QoL: no effect on patient's life (0-1); little effect (2-5); moderate effect (6-10); large effect (11-20); and extremely large effect (21-30).

Over a two-week period that included six field days, we surveyed participants in seven different rural and peri-urban communities (Figure 1) in the Atwima Nwabiagya North District outside of the urban setting of Kumasi, Ghana, where there is a large teaching hospital and few dermatology 
clinics. With the help of community liaisons and interpreters, research assistants solicited a convenience sample of adult individuals in the public market and residential areas to participate in a public health study regarding skin disease. The participants were recruited in a door-to-door fashion and were consented orally in their preferred language (Twi or English) if they decided to participate. Less than 10 individuals declined to participate. The research assistants read the questions out loud to participants then recorded their answers on the digital tablets. Community liaisons helped explain questions when needed. Individuals who responded positively to having a skin problem during the previous week received a specific identification number and were then privately examined by a dermatology resident and/or local dermatologist, often in the participants' own homes. After obtaining additional permission, high-quality photographs were taken of relevant skin findings using a standardized background. Two individuals refused to be photographed. While our role was primarily that of researchers, the dermatology resident (CWL) did offer medical advice to participants limited to diagnosis and medications that could be purchased safely over-the-counter. When the local dermatologist (MA) with a Ghanaian medical license was present, he often prescribed medications. If the patient required dermatologic care beyond what was feasible at local medical clinics, we arranged followup with the local dermatologist to his clinic in Kumasi. No participant required urgent medical attention.

Diagnoses were rendered on clinical appearance and history alone. Skin problems were categorized by the dermatology resident and local dermatologist based on the American
Academy of Dermatology's Burden of Skin Disease Categories ${ }^{15}$ with few adaptations to the categories (new categories added for "scabies" and "other"; categories for fungal infection and herpetic infection were separated). After the field days were complete, a board-certified dermatologist reviewed the photographs blinded to the diagnosis and also assigned a diagnostic category. Any diagnostic discrepancies $(<5 \%)$ were discussed and a consensus diagnostic category was reached. Discrepancies primarily came from determining which of the participants skin problems shown in the photographs was their primary skin complaint for which they answered the survey questions. For example, in one case the participant was concerned about scarring, rather than acne, and this had to be clarified to the blinded dermatologist reviewing the photos.

Patient demographics were summarized by descriptive statistics (means with standard deviations and frequencies with percentages, as appropriate), overall and by disease burden using DLQI. Differences were assessed using $t$-tests for continuous variables and chi-squared tests for nominal variables. All analyses were performed using SAS software version 9.2, copyright March 2008, SAS Institute Inc., Cary, NC, and significance was considered as $p<$ 0.05 .

\section{RESULTS}

Demographics and Dermatologic History Over six field days, 230 adult participants were surveyed. Participants were mostly female $(157 / 230 ; 68 \%)$, with a mean age of 41.6 years, employed (166/230; 72\%), married (111/230; 48\%), and had completed primary school education (127/230; $55 \%$ ). Distances lived from the district's major 
urban center ranged from 16.1 to 43.1 kilometers $(\mathrm{km})$ (mean $25.3 \mathrm{~km}$ ). Based on survey answers, $51 \%(117 / 230)$ reported a skin problem in the previous week.

\section{Dermatology Life Quality Index (DLQI) Results}

The DLQI was administered to all 230 participants who were surveyed. Of the 113 who did not report a skin disease in the previous week, $100 \% \quad(113 / 113)$ appropriately scored 0 points on the DLQI, which serves as a control group and helps validate this tool in this population. Of those who reported a skin disease in the previous week, $30 \%(35 / 117)$ reported no effect on QoL, 33\% (39/117) reported a little effect, $19 \%(22 / 117)$ reported a moderate effect, $16 \%(19 / 117)$ reported a large effect, and $1.7 \%(2 / 117)$ reported an extremely large effect. Among all participants, we compared those who reported little or no QoL burden (DLQI $\leq 5, n=187$ ) to those who reported moderate, large, or extremely large QoL burden ( $D L Q \mid>5, \quad n=43$ ) by demographic factors. Age, employment, education or marital status were not associated with QoL burden. Females and those living further from the city center were more likely to report at least moderate skin disease burden $(p \leq 0.02)$.

\section{Skin Disease Categories}

Of the 117 participants who reported a current skin problem in the previous week, 98 had a skin exam performed, 18 left the study area before a skin exam was performed, and 1 refused a skin exam. The most common skin problems identified were acne $(16 \%, 16 / 98)$, bacterial infection $(14 \%$, 14/98), pruritus (defined as participant itching without a rash) $(12 \%, 12 / 98)$, benign neoplasms/scars/cysts $(11 \%, 11 / 98)$, superficial fungal infections $(10 \%, 10 / 98)$, eczema $(8 \%, 8 / 98)$, hair and nail disorders $(7 \%, 7 / 98)$, and scabies $(5 \%, 5 / 98)$ (Table
1). Less frequent skin problems included seborrheic dermatitis, warts and molluscum, urticaria, herpes labialis, and ulcers. Three patients reporting a skin problem in the previous week had resolution of their skin findings or symptoms at the time of the exam, thus no diagnosis could be rendered. Although this study was not powered to detect any differences by skin disease category and QoL burden, clear differences existed in burden for some disease categories (Table 1). Acne (mean DLQI 2.9) and benign neoplasms and scars (mean DLQI 3.2) were less burdensome overall than bacterial infections (mean DLQI 8.6), dermatitis (mean DLQI 8.8), and ulcers (mean DLQI 9.6). Within our population, scabies infestation had the highest QoL burden (mean DLQI 15.6).

To provide a framework for skin disease severity, we categorized each participant's diagnosis by the level of management that would be initiated had the participant presented to a dermatology clinic. Treatment categories included counseling or reassurance, topical medication on the WHO essential medications list, ${ }^{16}$ oral medication on the WHO essential medications list, or additional diagnostic studies recommended (biopsy or culture). Of the 98 participants with skin exams, $20 \%$ $(20 / 98)$ could have been initially managed with counseling or reassurance, $59 \%(58 / 98)$ with a topical medication, and $6 \%(6 / 98)$ with an oral medication. $11 \% \quad(11 / 98)$ of participant skin findings warranted an initial diagnostic evaluation with culture or biopsy (Table 1). No trend in mean DLQI by management category existed.

\section{DISCUSSION}

Skin disease in rural Ghana is common$51 \%(117 / 230)$ of a convenience sample in a 
Figure 1. Map of communities around the Atwima Nwabiagya North District in the Ashanti Region outside of Kumasi, Ghana. Distances are listed from each community to Kumasi's city center where the nearest dermatology clinics are located. Mean distance $=25.3$ kilometers $(\mathrm{km})$.

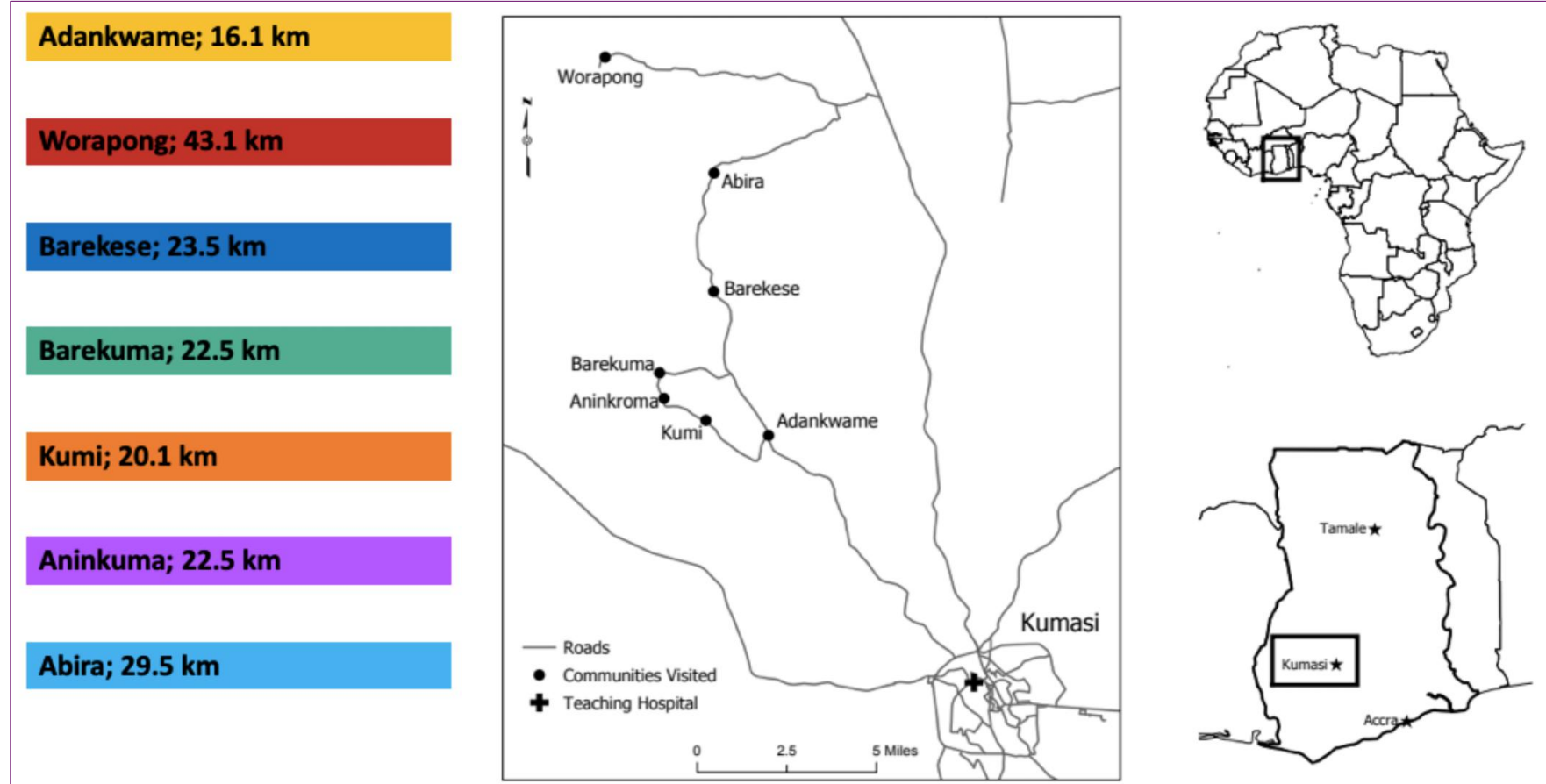

\section{BAREKESE SUBDISTRICT, ASHANTI REGION, GHANA}

Ghanaian community reported having a skin problem in the previous week. The most common diagnoses seen in our population were similar to those reported in the 2015 Global Burden of Disease study, ${ }^{1}$ in the South African study, ${ }^{14}$ and in a retrospective study of the urban dermatology referral center in Ghana. ${ }^{3}$

Skin-related QoL varied by gender in our cohort, with females endorsing a higher QoL impact. Women in rural Ghana tend to work in and around the home, and many of their skin diagnoses were related to frequent exposure to water and cleaning chemicals (e.g., paronychia, acral candidal, contact dermatitis), which may contribute to this increased burden.

Participants who lived further from a dermatology clinic were more likely to have at least moderate QoL impact from skin disease, suggesting that lack of access to care is a major obstacle to decreasing burden in underserved communities, and indeed remains a major barrier to providing adequate health care across all medical fields. Innovations like teledermatology are lauded as possible solutions to bring dermatologic care to these underserved populations. While communities closer to Kumasi had a clinic with infrastructure that could support teledermatology, even the most basic healthcare needs were not being met in more remote communities, including a functional health clinic with electricity and a reliable water source. Meeting basic healthcare needs in rural Ghana and other LMICs should be prioritized before improving access to specialty care.

Eighty percent of the skin problems evaluated in this population could have been treated with counseling/reassurance or a 
topical medication on the WHO essential medications list, ${ }^{16}$ and disease burden was not associated with treatment category. A recent study showed that training physician assistants in dermatology was an achievable method of expanding health care workers to rural populations in Ghana. ${ }^{5}$ Our study adds to the literature in that those with easily manageable skin conditions have a decreased QoL because of their skin disease. This suggests that better dermatologic education and availability of physician assistants would likely meaningfully decrease skin disease burden in these rural communities. For example, our participants with scabies had the highest mean QoL burden among all skin diagnoses made. Anecdotally, individuals and their households in the most rural of these communities have either traditionally tried kerosene to ameliorate scabies infestations, or suffer for months to years with this condition. Treatment of scabies with a topical scabicide and patient education performed by a trained physician assistant would be a welcome relief for the patients suffering from this curable condition.

As our cohort was a convenience sample of adults in rural Ghanaian communities, our prevalence figures are unlikely to be representative of the entire population. Individuals with skin disease may have been more likely to participate in the study, leading to potential overestimations of the skin disease burden in this population. Despite being a large study of skin disease impact on QoL in rural Africa, our study was insufficiently powered to perform skin disease-specific or multivariable analyses on factors associated with significant QoL impact. The DLQI tool, while linguisticallyvalidated, was not validated in this specific population beyond efforts taken to ensure participant understanding and it was not tested for reliability by contacting participants after the study to retake the survey. We minimized the limitations of a dermatology resident's diagnostic accuracy by involving a local dermatologist as frequently as possible and having an experienced board-certified dermatologist blinded to confirm diagnosis using highquality photographs.

Table 1. Skin diseases by modified American Academy of Dermatology's Burden of Skin Disease category, management category, and associated quality of life (QoL) burden as measured by the mean Dermatology Life Quality Index (DLQI).

\begin{tabular}{|c|c|c|}
\hline $\begin{array}{l}\text { Diagnostic Categories } \\
(n=98)\end{array}$ & $\begin{array}{l}\mathbf{N} \\
(\%)\end{array}$ & $\begin{array}{l}\text { Mean } \\
\text { DLQI }\end{array}$ \\
\hline Total & $\begin{array}{c}98 \\
(100)\end{array}$ & 5.6 \\
\hline Acne & $\begin{array}{c}16 \\
(16.3)\end{array}$ & 2.9 \\
\hline Bacterial infection & $\begin{array}{c}14 \\
(14.3)\end{array}$ & 8.6 \\
\hline Pruritus & $\begin{array}{c}12 \\
(12.2)\end{array}$ & 5.3 \\
\hline $\begin{array}{l}\text { Benign } \\
\text { neoplasm/Scar/Cyst }\end{array}$ & $\begin{array}{c}11 \\
(11.2)\end{array}$ & 3.2 \\
\hline Fungal & $\begin{array}{c}10 \\
(10.2)\end{array}$ & 5.1 \\
\hline Eczema/Dermatitis & $8(8.1)$ & 8.8 \\
\hline Hair/Nail Disorders & $7(7.1)$ & 4.7 \\
\hline Scabies & $5(5.1)$ & 15.6 \\
\hline Other & $4(4.1)$ & 3.5 \\
\hline Ulcers & $3(3.1)$ & 9.6 \\
\hline Seborrheic Dermatitis & $2(2.0)$ & 5 \\
\hline $\begin{array}{l}\text { Human Papilloma } \\
\text { Virus/Molluscum }\end{array}$ & $1(1.0)$ & 1 \\
\hline Urticaria & $1(1.0)$ & 3 \\
\hline Herpes infection & $1(1.0)$ & 0 \\
\hline No skin findings on exam & $3(3.1)$ & $\mathrm{n} / \mathrm{a}$ \\
\hline \multicolumn{3}{|l|}{$\begin{array}{l}\text { Treatment Categories } \\
(n=98)\end{array}$} \\
\hline Counseling/reassurance & $\begin{array}{c}20 \\
(20.4)\end{array}$ & 4.0 \\
\hline Topical treatment & $\begin{array}{c}58 \\
(59.2)\end{array}$ & 8.4 \\
\hline Oral treatment & $6(6.1)$ & 2.8 \\
\hline $\begin{array}{l}\text { Needs biopsy, culture, or } \\
\text { additional work-up }\end{array}$ & $\begin{array}{c}11 \\
(11.2)\end{array}$ & 5.9 \\
\hline
\end{tabular}

September 2020 Volume 4 Issue 5 


\section{CONCLUSION}

Adults in rural Ghana commonly suffer from a skin disease that impacts their quality of life, the vast majority of which are not medically complicated dermatologic conditions, but rather from diagnoses that could be managed with education and topical therapies. This study emphasizes that providing access to basic dermatologic care would address the vast majority of the skin disease burden in this community. Innovation and implementation of new technologies, while crucial in solving many public health problems, should not replace providing the most basic healthcare needs in underserved low- and middle-income communities. Future studies should test best practices for addressing unmet dermatologic needs of this population and measuring the subsequent impact on quality of life.

Acknowledgements: We acknowledge Dr. Clive Liu and Dr. Mark Herron for their financial support of this research project through the University of Utah Resident Alumni Research Fund. CWL expresses appreciation to Drs. Ty Dickerson, Scott Benson, and Rebecca Buxton for the invitation to conduct this research project in the context of their long-standing relationships with the Atwima Nwabiagya North District, KNUST, and KATH. We are grateful for the research assistants from KNUST, KATH and the University of Utah Division of Public Health who administered the surveys. We would like to thank the community liaisons who assisted us on the ground in participant surveys. This investigation was supported by the University of Utah Population Health Research (PHR) Foundation, with funding in part from the National Center for Research Resources and the National Center for Advancing Translational Sciences, National Institutes of Health through Grant 5UL1TR001067-05 (formerly $8 U L 1 T R 000105$ and UL1RR025764). Dr. Secrest is supported by a Dermatology Foundation Public Health Career Development Award. A special thanks to Nora Fino, MS, for statistical support.

\section{Conflict of Interest Disclosures: None}

Funding: Funding for this research came from an internal alumni research grant at the University of Utah Department of Dermatology
Corresponding Author:

Caroline W. Laggis, MD

30 North 1900 East, 4A330

Salt Lake City, Utah 84132

Phone: 843-870-1922

Fax: 801-581-6484

Email: caroline.laggis@hsc.utah.edu

References:

1. Karimkhani C, Dellavalle RP, Coffeng LE, Flohr C, Hay RJ, Langan SM, et al. Global Skin Disease Morbidity and Mortality: An Update From the Global Burden of Disease Study 2013. JAMA Dermatol. 2017;153(5):406-12.

2. Seth D, Cheldize K, Brown D, Freeman EF. Global Burden of Skin Disease: Inequities and Innovations. Curr Dermatol Rep. 2017;6(3):204-10

3. Rosenbaum BE, Klein R, Hagan PG, Seadey MY, Quarcoo NL, Hoffmann $\mathrm{R}$, et al. Dermatology in Ghana: a retrospective review of skin disease at the Korle Bu Teaching Hospital Dermatology Clinic. Pan Afr Med J. 2017;26:125.

4. Central Intelligence Agency. Ghana. In: The World Factbook. Washington, DC: Central Intelligence Agency; 2015. Accessed June 10, 2020 from

https://www.cia.gov/library/publications/theworldfactbook/geos/gh.html.

5. Truong A, Cobb NM, Hawkes JE, Adjase ET, Goldgar DE, Powell $\mathrm{DL}$, et al. Continuing Dermatology Education for Rural Physician Assistants in Ghana: An Assessment of Needs and Effectiveness. J Physician Assist Educ. 2018;29(1):19-24.

6. Finlay AY, Khan GK. Dermatology Life Quality Index (DLQI)--a simple practical measure for routine clinical use. Clin Exp Dermatol. 1994;19(3):210-6.

7. Ali F. Quality of life questionnaires. Accessed in October 2019 [Available from: https://www.cardiff.ac.uk/medicine/resources/quality-of-lifequestionnaires].

8. Abdel-Hafez K, Mahran AM, Hofny ER, Mohammed KA, Darweesh AM, Aal AA. The impact of acne vulgaris on the quality of life and psychologic status in patients from upper Egypt. Int $\mathrm{J}$ Dermatol. 2009;48(3):280-5.

9. Henok L, Davey G. Validation of the Dermatology Life Quality Index among patients with podoconiosis in southern Ethiopia. $\mathrm{Br} \mathrm{J}$ Dermatol. 2008;159(4):903-6.

10. Khoudri I, Lamchahab FZ, Ismaili N, Senouci K, Hassam B, Abouqal R. Measuring quality of life in patients with psoriasis using the Arabic version for Morocco of the Dermatology Life Quality Index. Int J Dermatol. 2013;52(7):795-802.

11. Kiprono S, Chaula B, Makwaya C, Naafs B, Masenga J. Quality of life of patients with vitiligo attending the Regional Dermatology Training Center in Northern Tanzania. Int J Dermatol. 2013;52(2):191-4

12. Klis S, Ranchor A, Phillips RO, Abass KM, Tuah W, Loth S, et al. Good quality of life in former Buruli ulcer patients with small lesions: long-term follow-up of the BUROLICO trial. PLoS Negl Trop Dis. 2014;8(7):e2964.

13. Wiese $S$, Elson L, Feldmeier $H$. Tungiasis-related life quality impairment in children living in rural Kenya. PLoS Negl Trop Dis. 2018;12(1):e0005939.

14. Jobanputra R, Bachmann M. The effect of skin diseases on quality of life in patients from different social and ethnic groups in Cape Town, South Africa. Int J Dermatol. 2000;39(11):826-31.

15. Lim HW, Collins SAB, Resneck JS, Jr., Bolognia JL, Hodge JA, Rohrer TA, et al. The burden of skin disease in the United States. J Am Acad Dermatol. 2017;76(5):958-72 e2.

16. World Health Organization. 19th WHO Model List of Essential Medicines. Updated June 2019. Available from: https://www.who.int/medicines/publications/essentialmedicines/en 